Investigaciones Turísticas

ISSN: 2174-5609

\title{
Turismo y sostenibilidad. Una propuesta metodológica para el estudio de la sostenibilidad turística a escala municipal
}

\author{
Anna Torres Delgado \\ Escuela Universitaria de Hotelería y Turismo CETT-UB \\ Universidad de Barcelona \\ anna.torres@cett.cat \\ Departamento de Geografía Física y Análisis Geográfico Regional \\ Facultad de Geografía e Historia de la Universidad de Barcelona \\ annatorres@ub.edu
}

\section{RESUMEN}

La actividad turística debe ser una práctica sostenible que contribuya al desarrollo económico, la equidad social, la revalorización cultural y la preservación del entorno. Con este objetivo la tesis afronta una de las principales dificultades en la aplicación real de la sostenibilidad en el sector: la falta de límites de sostenibilidad turística. Y lo hace a través del desarrollo de una metodología basada en indicadores que se aborda a dos niveles complementarios: el primero con la aplicación de un sistema de 26 indicadores de sostenibilidad social, económica y ambiental en 20 casos de estudio de Cataluña, y el segundo con la construcción de un Índice de Sostenibilidad Turística (ISOST) elaborado a partir del análisis empírico realizado y con vocación de uso general.

El Sistema de Indicadores se calcula en municipios representativos de las diferentes realidades turísticas y territoriales de Cataluña, de manera que se identifican las variables clave en la sostenibilidad turística para posteriormente agregarlas en un Índice de Sostenibilidad Turística (ISOST). EI ISOST establece umbrales de sostenibilidad turística extrapolables a partir de los cuales definir el grado de sostenibilidad turística de cualquier municipio turístico, cosa que supone la traducción de la sostenibilidad turística a valores cuantificables a través de una fórmula definida. La metodología propuesta, por tanto, es un sistema de recogida y tratamiento de datos efectivo para una planificación y gestión adecuada del turismo a escala municipal, así como una herramienta para valorar y definir estrategias turísticas de futuro y sostenibilidad para las destinaciones.

\section{Palabras clave}

Sostenibilidad turística, Indicadores, Sistemas de Indicadores, Índices, Municipios turísticos 


\title{
Investigaciones Turísticas \\ ISSN: 2174-5609
}

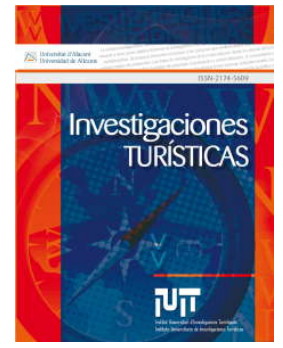

\section{Tourism and Sustainability: a Methodological Approach for the Study of Tourism Sustainability at the Municipal Level}

\author{
Anna Torres Delgado \\ University School of Hotel Management and Tourism CETT-UB \\ University of Barcelona \\ anna.torres@cett.cat \\ Department of Physical Geography and Regional Geographical Analysis \\ Faculty of Geography and History of the University of Barcelona \\ annatorres@ub.edu
}

\begin{abstract}
Tourism needs to be a sustainable practice that contributes to economic development, social equality, an increased appreciation of culture and the preservation of the environment. With this aim, the present thesis addresses one of the main difficulties faced in the real application of sustainability in the sector: the lack of boundaries around tourism sustainability. The thesis responds to this lack by developing a methodology based on indicators that work on two complementary levels: the first applies a system of 26 indicators of social, economic and environmental sustainability in 20 case studies in Catalonia, while the second, based on the empirical analysis, involves a Tourism Sustainability Index (ISOST) intended for general use.

The system of indicators is calculated for municipalities that are representative of Catalonia's varied tourism and territorial realities. It identifies the key variables of tourism sustainability and then aggregates them into the Tourism Sustainability Index (ISOST). ISOST extrapolates thresholds of tourism sustainability that can then be used to define the degree of tourism sustainability for any municipality participating in tourism. This involves using a defined formula to translate tourism sustainability into quantifiable ratings.

The proposed methodology, therefore, is a data collection and treatment system that is effective for adequate tourism planning and management at the municipal level, providing a tool for tourist destinations to assess and define their tourism strategies for the future with sustainability in mind.
\end{abstract}

\section{Key words}

Tourism sustainability, indicators, system of indicators, indexes, tourism municipalities 


\section{PLANTEAMIENTO DE LA INVESTIGACIÓN}

Hoy en día nadie pone en duda que la actividad turística debe ser una práctica sostenible que contribuya al desarrollo económico, la equidad social, la revalorización cultural y la preservación del entorno. Sin embargo, conseguir un turismo sostenible no es fácil, sobre todo teniendo en cuenta que la imprecisión del concepto dificulta su aplicación (López Palomeque, 2007). Se debe pues, aclarar el concepto y dotarlo de instrumentos que permitan transformar la idea teórica en valor práctico, es decir, en una serie de parámetros identificables y cuantificables. Con este paso el turismo sostenible debe dejar de ser una estrategia general, con muchas posibles interpretaciones y actuaciones, y convertirse una realidad alcanzable y adaptada a las circunstancias específicas de cada lugar. Es necesario desarrollar metodologías de identificación y cuantificación de impactos del turismo para facilitar la incorporación de la sostenibilidad en los procesos de decisión y gestión.

Pero si la definición de sostenibilidad turística implica dificultades prácticas y conceptuales importantes, no es menos su cuantificación. Aparte de las dificultades que surgen con respecto a la disponibilidad o generación de datos (White et al., 2006), el obstáculo más importante es establecer unos umbrales claros a partir de los cuales una actividad se considera sostenible o no. Esta reflexión se complica al pensar la diversidad de territorios, actores, recursos, intereses, etc. que confluyen en una actividad tan transversal y polifacética como el turismo. No hay un único modelo de desarrollo turístico universalmente correcto, sino que cada ámbito tiene unas necesidades sociales, cualidades territoriales y objetivos económicos diferentes a tener en cuenta en la construcción colectiva de alternativas sostenibles. Por ello, no se puede hablar en términos de sostenibilidad absoluta sino de tendencia a partir de un equilibrio entre la diversidad de situaciones locales y unos referentes (comparativos, temporales o científicos) que orienten la actividad bajo criterios de sostenibilidad.

Por tanto, la complejidad de la sostenibilidad turística dificulta que haya una metodología para su cuantificación universal y unánimemente aceptada. Y aunque existen varias propuestas de indicadores, todavía no se dispone de una metodología sintética y global que pueda extrapolarse a diferentes territorios o economías y además agregue variables de la sostenibilidad en un único índice (Pulido Fenández, 2007).

Precisamente en esta carencia es en la que se inspira esta investigación, fijando como principales objetivos la elaboración de un procedimiento metodológico concreto para el estudio de la sostenibilidad turística a escala municipal, y la definición de unos límites empíricos que permitan la clasificación de municipios en función del grado de sostenibilidad turística. Estudiar la sostenibilidad turística a escala municipal tiene la virtud de identificar los problemas y oportunidades de la actividad turística en el territorio concreto y en relación a las comunidades locales en que se desarrolla (Vera y Ivars, 2003; Chris Choi y Sirakaya, 2006), y a la vez garantiza una mejor y más fácil aplicación de las estrategias de sostenibilidad gracias a su nivel administrativo más directo. De esta manera, la metodología debe proporcionar un marco operativo a los 
gestores turísticos para facilitar la incorporación de la sostenibilidad en procesos de decisión y gestión de las destinaciones turísticas.

\section{METODOLOGÍA}

Tras haber desarrollado en profundidad un marco teórico y fenomenológico sobre el turismo y la sostenibilidad, así como sobre los instrumentos para la identificación y cuantificación de la sostenibilidad turística, se propone un Sistema de Indicadores adaptado a la escala municipal. El Sistema inicial recoge un total de 30 indicadores económicos, socio-culturales y ambientales seleccionados según los objetivos del estudio y la disponibilidad de datos para su cálculo. Sin embargo, para dotar a esta primera propuesta metodológica de consenso científico y, a la vez, minimizar la subjetividad implícita en todo proceso de elección y organización de indicadores, se realiza una encuesta a 54 expertos a través del método Delphi. Los expertos que participan en dicho proceso han sido escogidos por su especialización en temas de desarrollo sostenible del turismo y por proceder tanto de ámbitos de la investigación como de la planificación y gestión turística.

La contrastación científica resulta en un Sistema definitivo (véase Tabla 1) mejorado y más ajustado que cuenta con 26 indicadores organizados según dos modelos conceptuales combinados: las dimensiones de la sostenibilidad y el modelo causal DPSIR, que le otorgan la perspectiva holística del desarrollo sostenible y a la vez funcionamiento sistémico causa-efecto. A partir de aquí se asegura su eficiencia y utilidad en el estudio de la sostenibilidad turística municipal aplicándolo a la realidad, para lo cual se seleccionan un total de 20 municipios turísticos de Cataluña. Para escoger los casos de estudio se han tenido en cuenta los aspectos recogidos en la legislación para la declaración de un municipio turístico, pero también se ha otorgado especial importancia a la naturaleza geográfica de la actividad y a la realidad territorial resultante. De esta manera, no sólo se ha conseguido incluir los municipios con una estructura turística desarrollada y consolidada, sino también el equilibrio territorial entre las diversas regiones turísticas así como la variedad en las modalidades desarrolladas. En definitiva, los municipios seleccionados son representativos de las diferentes realidades turísticas y territoriales de Cataluña.

La aplicación práctica del Sistema de Indicadores en los casos de estudio se realiza mediante una ficha tipo que permite la recogida y el tratamiento sistemático de los datos, de manera que también se facilita el análisis comparativo y de la adecuación de cada municipio a los principios de sostenibilidad turística. Posteriormente, la valoración conjunta de los resultados y un análisis de correlaciones permiten identificar cuáles son los indicadores clave en la sostenibilidad turística municipal, paso decisivo para aproximar el concepto a través de un índice global.

El Índice de Sostenibilidad Turística (ISOST) propuesto parte de 12 indicadores simples estructurados según las tres dimensiones de la sostenibilidad (véase Tabla 2). Para su construcción se normalizan los indicadores en función de la distancia a la media de la muestra, de manera que todas las variables quedan estandarizadas respecto a una misma distribución. Seguidamente, se agregan a partir de la media 
aritmética los indicadores simples en tres subíndices (sociocultural, económico y ambiental) y, a su vez, los subíndices en el índice ISOST, ponderando en cada caso las variables con pesos iguales. De esta manera se obtiene un valor único asimilable al grado de sostenibilidad turística de cada municipio de estudio. Precisamente, el índice ISOST calculado para la muestra seleccionada de municipios permite identificar comparativamente diferentes niveles de sostenibilidad turística y establecer unos límites extrapolables al respecto. A partir del ranquin que se establece entre los municipios según su valor ISOST, y considerando que la media de la muestra determina el punto de inflexión a partir del cual un municipio se considera "sostenible" o "poco sostenible", la distribución de los casos en quintiles identifica los niveles de sostenibilidad turística.

\section{RESULTADOS}

La aplicación del Sistema de Indicadores en los casos de estudio ha resultado en el cálculo de 26 indicadores socio-culturales, económicos y ambientales para 20 destinaciones turísticas de Cataluña. Así pues, la generación de información para el análisis municipal de la sostenibilidad turística ha sido considerable, cosa que ha permitido un elevado nivel de profundidad analítica.

Sin embargo, el disponer de un volumen tan grande de información también ha limitado la interpretación integrada de los resultados, es decir, la valoración global de la sostenibilidad turística para cada municipio. En este sentido, el cálculo del índice ISOST ha suplido esta deficiencia proporcionando un único valor adimensional representativo de cada municipio. De acuerdo con la metodología expuesta anteriormente, el índice ISOST establece cuatro niveles de sostenibilidad turística

- ISOST $\geq 0,3=$ Municipio más sostenible turísticamente

- $0,3>$ ISOST $\geq 0=$ Municipio sostenible turísticamente

- $0>$ ISOST $>-0,3=$ Municipio poco sostenible turísticamente

- ISOST $\leq-0,3=$ Municipio muy poco sostenible turísticamente

La aplicación de estos límites en los 20 casos de estudio de Cataluña se resume en el Gráfico 1, y han identificado de los casos los municipios de Sitges y La Vall de Boí como los más sostenibles turísticamente, mientras que Piera y Mediona como los que menos. Teniendo en cuenta que la sostenibilidad turística calculada por el ISOST considera tanto variables ambientales como económicas y sociales, que Mediona y Piera obtengan valores bajos en el índice se explica por un turismo incipiente que aún no cuenta con una implicación realmente activa de los actores de territorio ni con una estructuración de la oferta y los recursos efectiva. Sitges y La Vall de Boí, en cambio, han logrado un desarrollo más equilibrado de la actividad, haciéndola rentable económicamente, pero también integrada en el territorio, responsable y equitativa socialmente, por lo que obtienen una mejor puntuación. 


\section{CONCLUSIONES}

La principal contribución científica de la tesis es precisamente el proceso metodológico desarrollado para cuantificar la sostenibilidad turística. Teniendo en cuenta que no hay una metodología universalmente aceptada, ni siquiera un consenso sobre su construcción y aplicación, el Sistema de Indicadores y el índice propuesto suponen un avance en este sentido.

La metodología se argumenta tanto a nivel conceptual, a través de la búsqueda bibliográfica y de experiencias sobre el turismo sostenible y los instrumentos para su identificación y cuantificación; como a nivel práctico, mediante su aplicación en municipios representativos de las diferentes realidades turísticas y territoriales de Cataluña. Precisamente este ensayo ha supuesto una diferencia cualitativa respecto otras propuestas, puesto que ha permitido garantizar su mensurabilidad y funcionalidad en el estudio de los impactos del turismo. También ha constatado que, si bien el tratamiento de la información indicador a indicador proporciona un nivel de profundidad analítica valioso, es difícil la interpretación integrada de los resultados o establecer una valoración global de la sostenibilidad turística para cada municipio.

Este hecho justifica y avala la elaboración final del Índice ISOST para disponer de un resumen estadístico en forma de valor único y simplificado de la sostenibilidad turística municipal. De hecho, el Índice es el producto final que resulta del conocimiento generado en la investigación. EI ISOST nace con vocación de ser aplicable por lo que se prima una metodología sencilla pero consistente, que utilice datos realmente disponibles en los organismos estadísticos y aplique procedimientos matemáticos simples.

Así pues, el procedimiento metodológico propuesto en esta investigación se estructura en un Sistema de Indicadores propio y un Índice complementario que contribuyen a la materialización del concepto de Sostenibilidad Turística, es decir, a la generación de datos específicos que han de facilitar la toma de decisiones y la gestión turística a los gestores municipales. Precisamente esta función también los hace idóneos para definir estrategias turísticas de futuro y sostenibilidad para las destinaciones.

\section{BIBLIOGRAFIA}

CHRIS CHOI, H. Y SIRAKAYA, E. (2006): "Sustainability indicators for managing community tourism" en Tourism Management, no. 27, pp. 1274-1289.

LÓPEZ PALOMEQUE, F. (2007): "Planificación territorial del turismo y sostenibilidad: fundamentos, realidades y retos" en Anuario Turismo y sociedad, vol. VIII, pp. 51-68. Facultad de Administración de Empresas Turísticas y Hoteleras. Universidad de Colombia.

PULIDO FERNÁNDEZ, J.I. (2007): "Sostenibilidad de los destinos turísticos: Una aproximación a su medición en España" en López, D. y Pulido, J. I. (ed.): La actividad turística española en 2006, AECIT, pp. 247-263. Editorial universitaria Ramón Areces, Jaén. 
VERA, J.F. y IVARS, J.A. (2003): "Measuring Sustainability in a Mass Tourist Destination: Pressures, Perceptions and Policy Responses in Torrevieja, Spain" en Journal of Sustainable Tourism, vol. 11, no. 2-3, pp.181-203.

WHITE, V.; MCCRUM, G.; BLACKSTOCK, K.L.; y SCOTT, A. (2006): Indicators and Sustainable Tourism: Literature Review. The Macaulay Institute, Aberdeen (Escocia). 
Tabla 1. Sistema definitivo de indicadores de sostenibilidad turística.

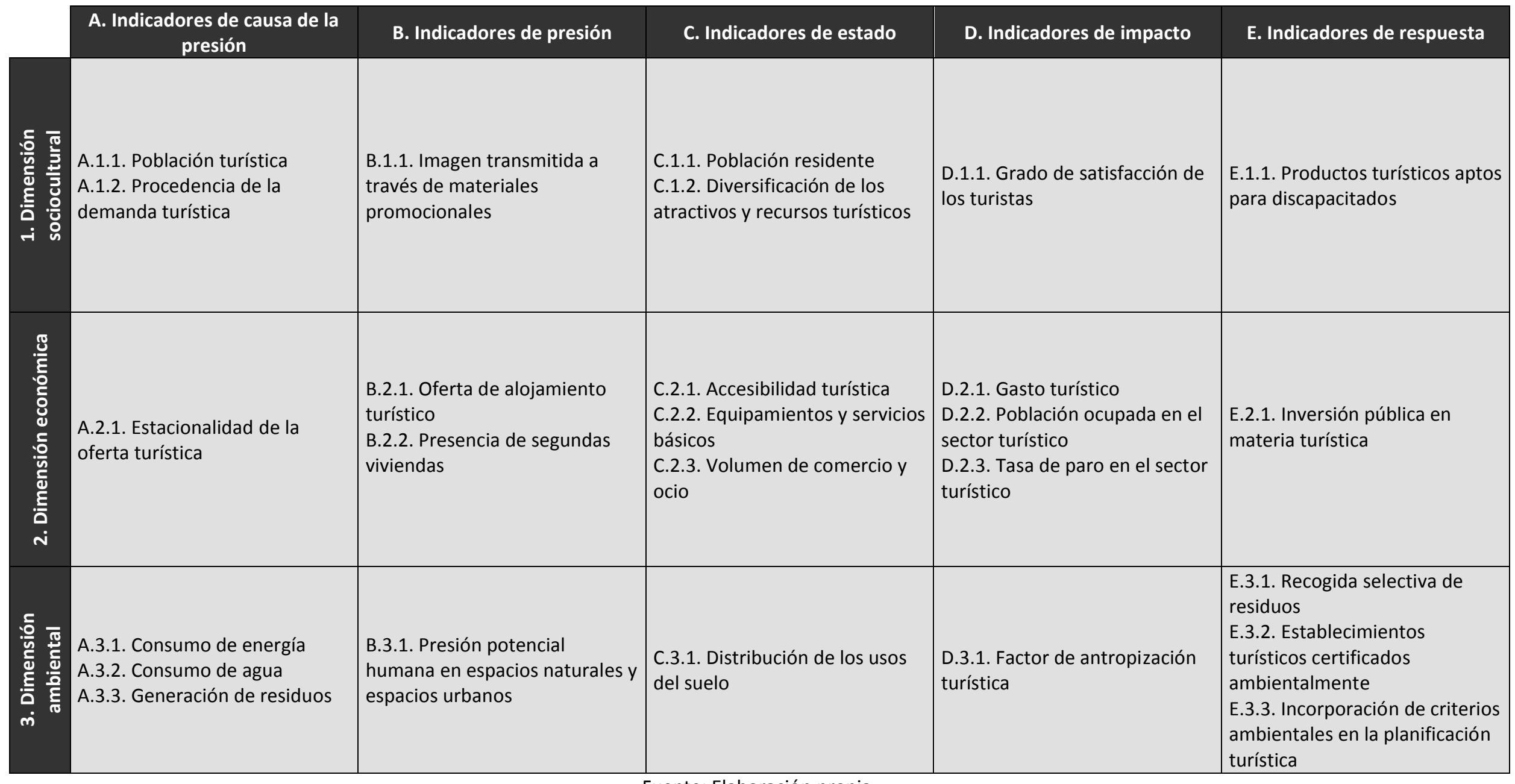

Fuente: Elaboración propia. 
Tabla 2. Indicadores simples definitivos para la elaboración del ISOST.

\begin{tabular}{|l|l|}
\hline \multicolumn{1}{|c|}{ Indicadores según dimensión } & \multicolumn{1}{c|}{ Cálculo } \\
\hline 1. Dimensión sociocultural & \% población Turística Equivalente \\
\hline A.1.1. Población turística & oo de diferentes categorías de recursos turísticos \\
recursos turísticos & $\begin{array}{l}\text { № de diferentes tipos de adaptaciones para } \\
\text { discapacitados }\end{array}$ \\
\hline $\begin{array}{l}\text { E.1.1. Productos turísticos aptos para } \\
\text { discapacitados }\end{array}$ & \% plazas turísticas disponibles (anual) \\
\hline 2. Dimensión económica & \% viviendas secundarias \\
\hline A.2.1. Estacionalidad oferta turística & \% de presupuesto en turismo \\
\hline B.2.2. Presencia de segundas viviendas & Consumo Kwh/Población Total Presente/día \\
\hline E.2.1. Inversión pública en turismo & Consumo litros/ Población Total Presente /día \\
\hline 3. Dimensión ambiental & Residuos Kg/ Población Total Presente /día \\
\hline A.3.1. Consumo de energía & \% suelo urbano \\
\hline A.3.2. Consumo de agua & $\begin{array}{l}\text { \% establecimientos de alojamiento turístico } \\
\text { certificados }\end{array}$ \\
\hline A.3.3. Generación de residuos & $\begin{array}{l}\text { № de planes turísticos municipales que incorporen } \\
\text { criterios ambientales }\end{array}$ \\
\hline C.3.1. Distribución de usos del suelo & $\begin{array}{l}\text { E.3.2. Establecimientos turísticos certificados } \\
\text { ambientalmente }\end{array}$ \\
\hline $\begin{array}{l}\text { E.3.3. Incorporación de criterios ambientales } \\
\text { en la planificación turística }\end{array}$ & Focion propia. \\
\hline
\end{tabular}

Fuente: Elaboración propia.

Gráfico 1. Municipios de estudio según el grado de sostenibilidad turística.

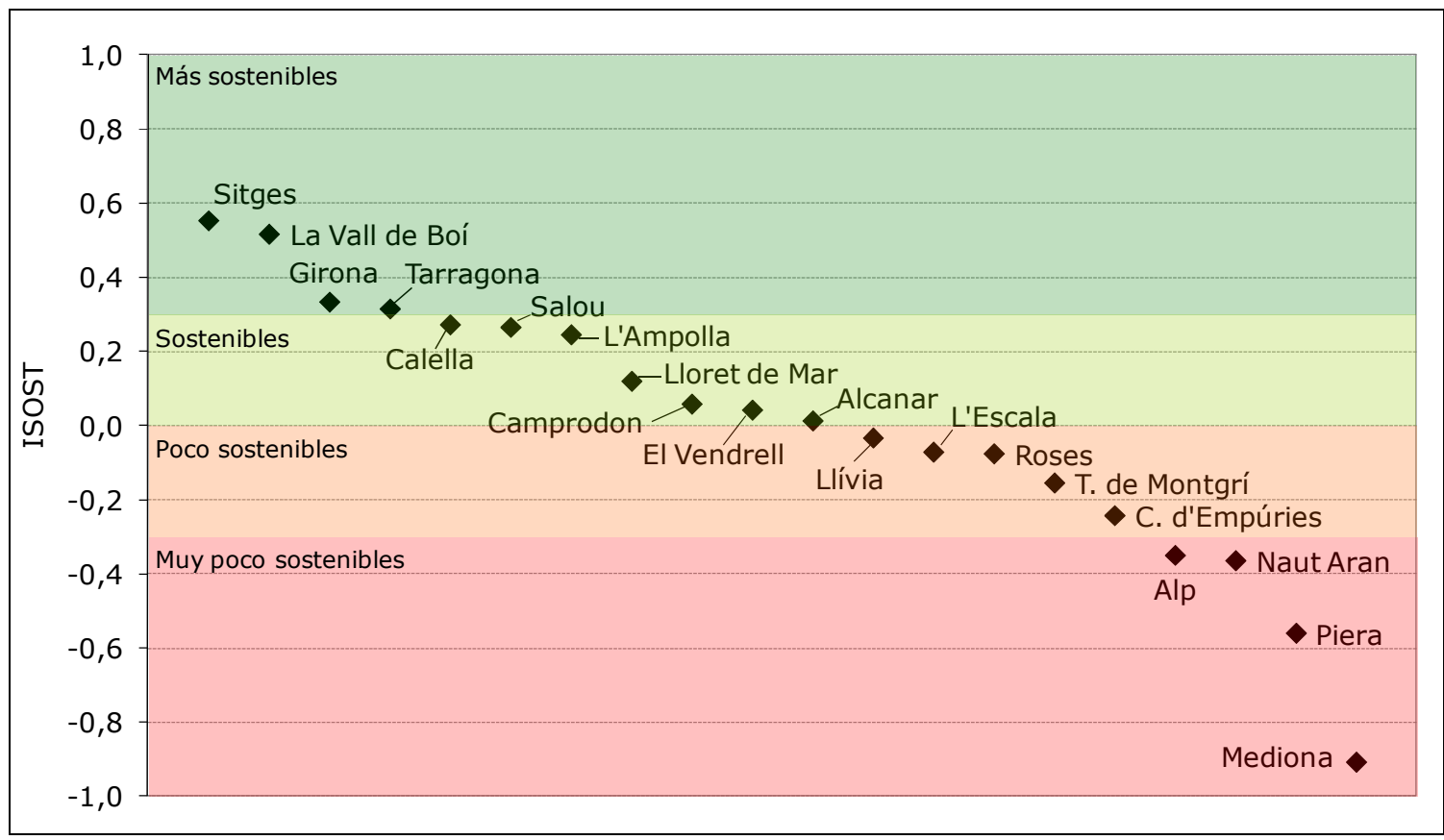

Fuente: Elaboración propia. 